\title{
Perspective on the economic evaluation of deep brain stimulation
}

\author{
Emma Sarah McIntosh* \\ Department of Public Health, Health Economics Research Centre, University of Oxford, Oxford, UK
}

\section{Edited by:}

Chiara Saviane, SISSA, Italy

Reviewed by:

Pablo Martinez-Martin, Fundación

Centro de Investigación de

Enfermedades Neurológicas, Spain

Ivar Sonbo Kristiansen, University of

Oslo, Norway

\section{${ }^{*}$ Correspondence:}

Emma Sarah Mclntosh, Department of Public Health, Health Economics

Research Centre, University of Oxford, Old Road Campus, Oxford OX3 7LF, UK. e-mail:emma.mcintosh@dphpc.ox. ac.uk
Parkinson's disease (PD) is an example of a disease area experiencing increasing use of deep brain stimulation (DBS) to treat symptoms. PD is a major cause of morbidity and has a substantial economic impact on the patients, their caregivers, the health service, and broader social and community services. The PDSURG Collaborators Group reported that DBS surgery for patients with advanced PD improves motor function and quality of life that medical therapy alone at 1 year but there are surgery related side effects in a minority (Williams et al., 2010). The aim of this paper however is to build upon the knowledge generated from evaluating DBS in PD and to provide a detailed perspective on the economic evaluation of DBS more generally with a view to providing a framework for informative design of DBS economic evaluations. This perspective will outline the key categories of resource use pertinent to DBS beyond the surgical scenario and into the broader aspects of follow-up care, adverse events, repeat procedures, social and community care, patient and carer costs, and will explore the importance of handling capital costs of DBS equipment appropriately as well as including costs occurring in the future. In addition, this perspective article will outline the importance of capturing broader aspects of "outcome" or benefits as compared to those traditional clinical measures used. The key message is the importance of employing a broad "perspective" on the measurement and valuation of costs and benefits as well as the importance of adopting the appropriate time horizon for evaluating the costs and benefits of DBS. In order to do this effectively it may be that alternative methods of economic evaluation in health care to the commonly used cost-effectiveness analysis may have to be used, such as cost-benefit analysis (Mclntosh et al., 2010).

Keywords: economic evaluation, deep brain stimulation, broad perspective, time horizon, Parkinson's disease

\section{INTRODUCTION}

The aim of this paper is to outline issues pertinent to the economic evaluation of deep brain stimulation (DBS) with a view to providing a general framework for future economic evaluations of DBS technology whatever the indication. The broad economic issues around DBS technology are currently an under researched topic. This is due to the lack of evidence from large comparative studies but also due to a lack of theoretical consideration of the key range of costs and benefits associated with DBS, their long-term impact and related methodological considerations. Whilst a number of economics publications have contributed to the evidence base in this area independently on costs and health-care outcomes in the area of Parkinson's disease (PD) significant gaps remain on the "cost-effectiveness" of DBS technology more generally. At present Government funding for many health-care procedures such as DBS is often based on inaccurate costs and narrow measures of benefit. This is partly due to the complexity of costing disease treatment pathways and limited vista on the definition of benefits used. DBS is no exception to this. It is therefore important to have robust evidence on both the short and long-term costs and health-care outcomes of DBS interventions. Such information allows decision makers to prioritize funding appropriately using sound evidence.
This perspective article will begin by introducing the key cost and outcome components relevant to DBS and this will be followed by specific topics of methodological importance in the DBS area related to both costs and benefits. Finally, a discussion will be based around the key economic factors that will influence the cost-effectiveness of DBS as well as the theoretical and methodological challenges to using a broad evaluation perspective, with particular reference to the importance of measuring outcomes in the DBS area.

\section{THE IDENTIFICATION OF RESOURCE USE DATA IN DBS}

A detailed comparison of patient resource utilization and costs of all aspects of DBS surgery and its comparator is crucial in any evaluation of DBS. Ideally such data will come from a randomized controlled trial (RCT) or other rigorous design. Resource use data in DBS are typically related to the resources outlined in Table $\mathbf{1}$.

\section{HOSPITAL STAY}

In DBS it is predicted that the duration of hospital stay for the DBS surgery and any related subsequent events and adverse events related to the surgery such as infections will be a significant key cost driver of the total cost. It is therefore important to identify hospital stay in the economic evaluation as accurately as possible. 


\section{DBS EQUIPMENT}

As outlined in Table 1 above the different types of equipment involved in DBS are: implantable pulse generator (IPG), electrodes, extension leads, patient controller as well as the large capital items used by the surgeons, nurses, and electrophysiologists during the operation such as the stereotactic frame which keeps the head still during the DBS operation and the planning station used to identify the appropriate coordinates in the brain for targeting the electrodes. There are a number of different suppliers producing these equipments and generally the prices are similar. Due to the high cost of these pieces of equipment it is important that they be accurately annuitized as a function of lifespan and cost per use estimated based on throughput. A paper by Joint et al. (2002) estimated the hardware-related problems of DBS and noted a $20 \%$ rate of hardware-related problems in their cohort as compared to a range of $7-65 \%$ reported by other groups. The cost of the equipment may change over time for instance in DBS a new implant has been developed with a much longer battery life however the capital cost of this is more

Table 1 | Resource data typical in DBS.

\begin{tabular}{|c|c|}
\hline Stage & Resource use \\
\hline \multirow[t]{2}{*}{ Pre-operative } & Clinic appointments \\
\hline & Pre-operative assessment: neurology staff \\
\hline \multirow[t]{7}{*}{ Operative } & Theater time: neurology staff and consumables \\
\hline & DBS equipment: implantable pulse generator (IPG); \\
\hline & electrodes; leads; extension leads; patient controller \\
\hline & Planning station \\
\hline & Stereotactic frame \\
\hline & Robotic equipment \\
\hline & Hospital stay (neurology ward) \\
\hline \multirow[t]{7}{*}{ Post-operative } & Follow-up clinic appointments \\
\hline & GP visits \\
\hline & PD nurse appointments \\
\hline & Subsequent procedures: theater time, \\
\hline & hospital stay, equipment \\
\hline & Adverse events: all operative resources outlined \\
\hline & above; medications (e.g., antibiotics for infections) \\
\hline All stages & Social and community care costs: GP visits; physiotherapy \\
\hline & visits; nurse visits; community psychiatric care visits \\
\hline \multirow[t]{6}{*}{ All stages } & Patient costs: out of pocket expenses; travel costs \\
\hline & attending appointments; medication costs; aids and \\
\hline & adaptations; modifications to homes; cleaner costs; \\
\hline & gardening costs; home equipment such as special \\
\hline & beds/shower units; Loss of Income due to time \\
\hline & off work/inability to work \\
\hline All stages & Informal carer costs: time spent caring for PD patient \\
\hline & (value of time); loss of income due to time spent caring \\
\hline \multirow[t]{5}{*}{ All stages } & Hospital stay \\
\hline & Institutionalization costs \\
\hline & Care home costs \\
\hline & Meals on wheels \\
\hline & Day centers \\
\hline All stages & Medication/drugs \\
\hline
\end{tabular}

expensive and currently in the UK only few patients have been given this option. Table 2 below gives a summary of the range of costs for the DBS equipment.

\section{MEDICATION}

It is the case in PD that often following DBS surgery patients become less reliant on certain forms of medication. The reduction in such a cost should be taken into consideration by the economic evaluation as a function of time. Drug prices should been calculated from the net cost used in pricing excluding VAT (For UK specific medication costs are obtained from the British National Formulary, BNF; Joint Formulary Committee, 2010. Prices should reflect the cost per individual patient dosage calculated on a daily basis and multiplied by the appropriate time period. In DBS data should also be collected on medication change over time to allow for the beneficial effects of DBS permitting a reduction in medication use. Such detailed information on drug use is important in the evaluation of DBS as it is the case that whilst the majority DBS costs are up front and occur at the time of the surgical episode it may be the case that the offset reductions in expensive drugs (e.g., Apomorphine in PD) may continue for a number of years and give rise to substantial cost savings which, if ignored, could bias the economic evaluation. In the situation where long-term data are unavailable then the use of economic modeling techniques and sensitivity analysis methods are recommended to identify the magnitude of savings.

\section{SERIOUS ADVERSE EVENTS}

In DBS in PD some of the more common serious adverse events arising following surgery include infections (often caused by breakthrough of electrodes), stroke, hemorrhage, DVT (Costs identified in Ramzi and Leeper, 2004) and fractures arising from falls following DBS (wrist, humerus, pelvic, odontoid). The main cost incurred with infections are the cost of hospital stay and antibiotic treatment but the costs increase markedly where the infection requires that the IPG and electrodes be removed and a new set inserted. Such adverse

Table 2 | Approximate costs" of DBS equipment and related planning equipment.

Item

Cost UK (2008)

Implantable pulse generator

$£ 7,000-£ 8,500$

Electrode

£800-£900*

Extension lead

£700-£850*

Patient controller

£600-£700

Accessory kit

£75-£100

Planning station

$£ 60,000^{1}$

Stereotactic frame

f75,000²

\#Costs provided are approximated based on averages from different suppliers and exclude VAT.

* Usual number required for DBS surgery $=2$.

1 Life span usually 5 years (this cost does not include maintenance costs not take into account throughput).

2 Life span usually 3 years (this cost does not include maintenance costs nor take into account throughput).

Source: personal correspondence with suppliers. 
events are likely to be similar across disease areas as they are common to the technology rather than the disease hence, the costing for such adverse events may be transferable across economic evaluations.

\section{INFORMAL CARE COSTS}

The number of carers per patient and the duration of time spent caring per week should be collected where possible so that the impact of DBS on such "informal care" can be estimated. The informal costs of caring can be estimated based on a number of different assumptions for the hourly rate of caregivers (Van den berg and Ferrer-I-Cabonell, 2007; De Meijer et al., 2010). Informal care can comprise a substantial part of long-term care and often substitutes formal home and nursing home care (Van Houtven and Norton, 2004). Van Houtven and Norton (2004) show that informal care reduces formal health-care use and delays nursing home entry. Informal care can be thought of as comprising elements such as home keeping, personal care, support with mobility, administrative tasks, and socializing. In health care, unlike the available unit costs identifiable for formal care such as those outlined in Table 2, market prices for such informal care services often do not exist. For example the cost of informal care may not reflect the true societal value of resources attributed to this activity (Drummond et al., 2005) and as such "shadow" prices or proxy values are used (McIntosh et al., 2010). With health services becoming increasingly reliant on informal care and the associated shift in costs from the health-care sector to the community, for instance through early discharge programs, the substitution of inpatient care with ambulatory care and the move toward community care of the mentally ill - the greater the importance attached to recognizing and valuing the true cost of unpaid inputs. Provision of informal care may also result in additional costs (although perhaps not direct financial costs within the health-care sector) which should also be incorporated into the value of the unpaid input. These additional costs are shown in Table 3. In DBS it may be the case that the amount of informal care required by recipients is reduced with successful DBS and as such should be incorporated into the economic evaluation.

\section{INSTITUTIONALIZATIONS AND CARE HOME COSTS}

Where DBS treatment allows patients to continue staying in their own home being cared for by their carers then the avoidance of institutionalization and care home costs can be substantial. It important therefore that such costs be included in the evaluation of DBS - it may be that this gives rise to substantial savings over time.

Table 3 | Additional costs associated with informal care.

\begin{tabular}{ll}
\hline Additional "costs" & "Shadow" price* \\
\hline Time spent traveling by patients, & Value of time \\
relatives, carers & \\
Time spent waiting for consultation, during & Value of waiting time \\
consultation, treatment, and rehabilitation & Opportunity cost of time \\
Leisure time lost (if time allocated & Value of leisure \\
to unpaid activity & activities forgone \\
involves a displacement of & \\
non-working time) & \\
\hline *Shadow prices are proxy values where there are no identifiable market prices \\
or values.
\end{tabular}

\section{PRODUCTIVITY COSTS AND DBS}

Productivity costs as defined by the Washington Panel are: “...costs associated with lost or impaired ability to work or engage in leisure activities due to morbidity and lost economic productivity due to death" (Gold et al., 1996). Brouwer et al. (2001) propose an alternative definition of productivity costs as "Costs associated with production loss and replacement costs due to illness, disability, and death of productive persons, both paid and unpaid." For a comprehensive guide to all three methods both theoretically and practically as well as a direct comparison of the methods see Pritchard and Sculpher (2000), see also (Rice and Cooper, 1967; Koopmanschap et al., 1997). Productivity cost are pertinent to DBS as this technology may enable patients to engage in or indeed return to an economically productive life such as returning to a job or engaging in valued leisure time once again. As such any economic evaluations in the area of DBS should ensure that employment status or daily activities are measured accordingly so that the impact of DBS on such activities are captured.

\section{KEY CONCEPTS IN COSTING METHODOLOGY USED FOR DBS EVALUATIONS}

A "micro" approach to the costing of surgery and medical resources uses patient-specific data itemized by use of resources and such an approach is useful in early evaluation of new technologies where the key cost drivers have yet to be identified. The key cost drivers in DBS will ultimately be the high capital costs of the equipment which is patient-specific as well as the ward stay and follow-up procedures including adverse events and repeat implantations. It is recommended that where DBS is being evaluated for the first time in a disease area a micro-costing approach be used to identify the key cost drivers.

\section{BASE YEAR}

It is important to identify all costs in the same base year. This may require inflation or deflation of some items. The current discount rate for annuitization of capital items is $3.5 \%$ as recommended by HM Treasury (HM Treasury, 2003). Bearing in mind that to get to the final "total" cost figure many different variables have to be added together and different data points will have differing levels of complete data from patients hence missing data should be analyzed using appropriate techniques such as multiple imputation methods (Van Buuren et al., 1999).

\section{EQUIVALENT ANNUAL COST (EAC) OF DBS EQUIPMENT COSTS}

As outlined in earlier annuitization is an important costing method relevant to DBS as a result of the high cost items of equipment used. Capital costs tend to occur at a single point in time however, capital assets are used over time and can be sold at any time therefore the opportunity cost of capital is spread over time. As a consequence of this, the appropriate costing of capital items requires the calculation of an EAC. This EAC is therefore the capital cost apportioned into EACs as a function of expected lifespan and appropriate discount rate. In addition to this however to obtain a "unit cost per use" items of capital generally also require the inclusion of annual servicing and replacement part costs and these "annual costs" should then be divided by the annual throughput of patients using the equipment to 


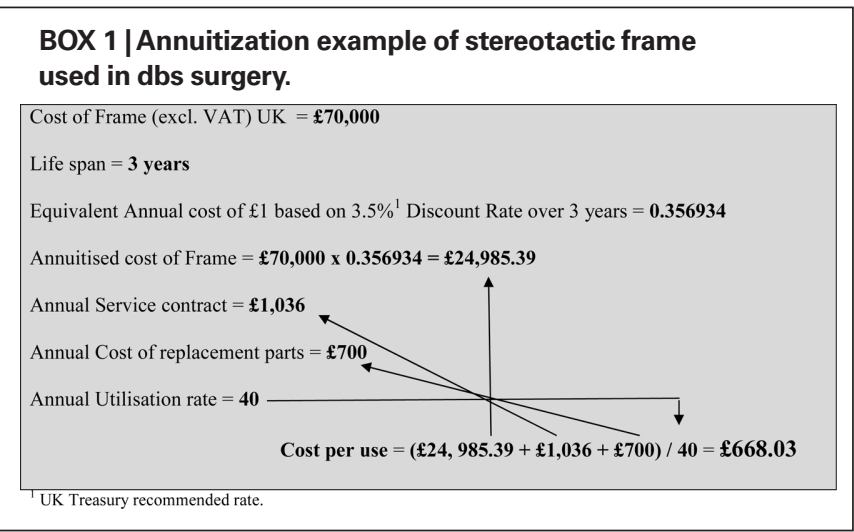

obtain a unit cost per use. Box 1 below provides a worked example of the annuitization of the cost of a stereotactic frame and this annuitized cost transformed into a "cost per use" as a function of annual service costs, annual replacement parts, and annual utilization rate.

\section{HANDLING UNCERTAINTY IN DBS ECONOMIC ANALYSES}

Briggs (2001) distinguishes among a number of different types of uncertainty depending upon whether the data are patient level or from decision analytic models. In stochastic analyses such as alongside clinical trials they identified four main types of uncertainty: methodological; sampling variation; extrapolation; and generalizability/ transferability. For all types of uncertainty apart from extrapolation, where modeling methods are recommended, sensitivity analysis is the recommended approach to handling uncertainty. Sensitivity analysis is a method whereby various parameters in the analysis are varied in order to test the impact on the overall result. The main types of sensitivity analysis are one way; multi-way; scenario analysis, threshold analysis, and probabilistic sensitivity analysis (PSA). See Briggs (2001), Drummond et al. (2005), and Glick et al. (2007) for fuller expositions of these methods. Key parameters influencing the cost of DBS and which may make the total cost variable "sensitive" to change and therefore impact the overall result of the study include the following: source of unit costs; cost perspective; lifespan of capital items such as those outlined in Table 2; patient throughput; discount rate and annual service and maintenance costs. Box 1 above outlined the importance of appropriate methodology to identify the cost per use of a stereotactic frame used in DBS. Box 2 below now uses this to highlight the importance of sensitivity analysis on key costs in DBS based on changing the key assumptions regarding lifespan, throughput, and annual service and maintenance costs.

Box 1 above showed that the base cost of $\mathfrak{E} 668.03$ per use was estimated based on the following assumptions: lifespan $=3$ years; annual service cost $=\mathfrak{E} 1,036$; annual cost of replacement parts $=\mathfrak{E} 700$ and annual utilization rate $=40$. Carrying out the sensitivity analysis outlined in Box 2 shows that the cost per use is sensitive to changes in lifespan and throughput but less sensitive to changes in annual service and maintenance costs. This analysis shows that for the stereotactic frame cost involved in DBS, efficiency savings could be achieved by increasing its lifespan (albeit incurring some extra maintenance and service costs) and increasing the annual throughput of the Frame - this may be achieved by diversifying the frame's use to procedures other than DBS.

\section{BOX 2 | Sensitivity analysis of DBS stereotactic frame cost assumptions.}

\section{Assumption}

Costperuse(f)

Lifespan of 5 years

Lifespan of 10 years

$£ 430.99$

$£ 253.82$

Annual throughput of 20 patients

Annual throughput of 80 patients

Reducing the annual service and

$£ 1,336.07$

$£ 334.02$

$£ 646.33$

\section{THE IMPORTANCE OF TIME HORIZON WHEN EVALUATING DBS TECHNOLOGIES}

In the UK the National Institute for Health and Clinical Excellence (NICE) makes recommendations to the NHS on new and existing medicines, treatments, and procedures. NICE have devised the concept of a reference case which was developed by experts in the methodological aspects of economic evaluation. The reference case, based on that published by Gold et al. (1996) specifies the methods considered by the institute to be consistent with an NHS objective of maximizing health gain from limited resources. Within this reference case the time horizon recommended should be long enough to include all relevant costs and outcomes relating to the intervention. This is an importance issue for economic evaluation of DBS technologies as it is the case that many of the expensive equipment and hospital stay costs are very much "up front" and it is possible that the savings in terms of reduced medication and other health service costs do not occur till further in the future. Likewise additional costs incurred such as replacement IPGs and adverse events related to for example, infection, hemorrhage, and falls may not happen till later then these costs must be included in the economic evaluation to reveal the true economic picture of DBS in relation to the next best alternative treatment.

\section{IDENTIFICATION, MEASUREMENT, AND VALUATION OF HEALTH ECONOMIC OUTCOMES IN DBS}

While the majority of this paper so far has been dedicated to the identification, measurement, and valuation of resources involved in DBS another crucial element to any economic evaluation is the identification, measurement, and valuation of outcomes. Indeed, it is my opinion that this is an area under researched and of great significance to the economic research arena in this area. Investigation into the science behind outcomes valuation is not always considered by many to be the job of economists who are often heralded as "accountant" types however this is far from true. Indeed mainstream economists in many disciplines from environmental to transport and health economics have developed sound theoretical methodologies for valuing outcomes that are increasingly being used.

\section{MEASURING HEALTH OALYs AND BEYOND}

The subject of measuring health and disease is the concern of many disciplines beyond health economics (Bowling, 1991, 1995), including public health, epidemiology, and statistics. In health economics it is widely accepted that it is theoretically possible to use numeraires such as health state utility to value outcomes (Torrance and 
Sackett, 1972; Torrance, 1976; Sackett and Torrance, 1978; Torrance et al., 1982). Culyer (1989) argued for an "extra welfarist" approach to health. Unlike the traditional economic "welfarist" approach which measures everything deemed to impact upon a person's overall utility (including health) the task of measuring only changes in "health" was advocated in the "extra welfarist" approach, with the quality adjusted life year (QALY) as the instrument of choice (Williams, 1985; Culyer, 1989). As a consequence, much of the health economics literature in recent years has concentrated on issues around measuring and valuing preferences for health care in non-monetary mediums, i.e., quality of life (QOL; Drummond et al., 1987; Buckingham, 1993, 1995; Richardson, 1994). This has led to the development of health state valuation measures such as QALYs (Williams, 1985). In health economics the QALY is the common outcome measure employed by health economists alongside the many clinical outcomes measures specific to disease areas (such as the Hoehn and Yahr, 1967 and PDQ-39 Jenkinson et al., 2008 in PD) and measures of clinical effectiveness commonly used in costeffectiveness analyses (Drummond et al., 2005). The advantage of the generic QALY is its ability to be compared across many disease areas allowing cost-utility estimates to be compared. In DBS surgery, given the global acceptance of the QALY as a generic measure of QOL (Dolan et al., 1995), with its 150 language translations, it would be recommended as a generic measure to be administered alongside disease specific measures of relevance to the particular condition. The EuroQol Group launched a new EQ-5D-5L (EQ5D 5 level) self-complete version in 2009 with the aim of further improving the sensitivity and reducing ceiling effects of the existing EQ-5D-3 level version. This is now available in more than 40 translations. Future plans include EQ-5D-5L versions in web and tablet format (The EuroQol Group, 2011).

\section{RISKS IN DBS}

As outlined earlier, there are a number of risks associated with DBS surgery and how these are communicated effectively to patients is important. It is often the case with DBS surgery, such as in advanced PD that DBS surgery is the only remaining option once medication effects have worn off over time. In this situation, for some patients, there are little viable alternative treatments. The risks and benefits of the surgery must be explained to the patient, often using detailed patient information leaflets summarizing the available evidence on the risks, side effects, and long-term outcomes of the procedure. The University of Florida Movement Disorders Centre (MDC; Movement Disorders Centre UoF, 2011) outline the main risks occurring during the surgical procedure. One of these risks occurs when microelectrodes are inserted into the brain to determine the best target location. If a microelectrode, or alternatively the DBS lead, punctures a blood vessel it can lead to a stroke or a strokelike syndrome which may result in weakness, numbness, sensory loss, visual difficulties, or a host of other neurological problems. Additionally, patients with cognitive dysfunction may worsen following DBS, and the surgery may affect one of many mood and cognitive circuits leading to changes such as depression, laughter, memory problems, or other psychiatric, and/or behavioral features. Additionally, there is a chance the lead may migrate, or the electrode, connecting wire, or implanted pulse generator may break and need to be replaced. Any time a foreign body is implanted into a human there is a risk for infection, both at the skin level, and in the brain. The batteries in the device will have to be changed requiring additional surgery, with the average time to battery replacement differing based on the underlying disorder, as well as the stimulation settings. As with all surgical procedures, there is a small chance of infection, and death from the procedure. To download the patient information leaflet used in the DBS in PD study (PDSURG) to see how the risks of DBS surgery were described in this context please see the PDSURG website (http://www.pdsurg.bham.ac.uk).

Clinicians have seen first-hand how DBS has provided significant improvements in QOL for patients with PD, tremor, dystonia, and other movement and basal ganglia related brain disorders. However as outlined above DBS surgery is not suitable for everyone and clinicians we will need to be able to select the best possible candidates by making assessments as to those patients who have the most favorable risk-benefit ratios and delivering that information effectively (Movement Disorders Centre UoF, 2011). Clinicians will need to better educate patients about who is a suitable candidate, and what they can expect and anticipate from the DBS surgical approach to their problems. As outlined by the MDC perhaps the biggest risk of surgery is that for patients and families the surgery will not meet perceived expectations. Hence it is recommended that patients have an in depth consultation with a movement disorders neurologist to outline the risks and benefits of DBS. The MDC at the University of Florida offers a mnemonic device for PD patients interested in DBS to help educate them and alter their perceptions to more closely match what is known about anticipated benefits of surgery. The future however will also bring about changes in risks and benefits as the technology evolves. For instance there will be rechargeable devices as well as devices that will work on a closed-loop circuit (meaning they will automatically turn on when needed).

\section{THE IMPORTANCE OF BROAD OUTCOME MEASURES IN DBS}

In DBS the use of QALY's as a generic measure is recommended alongside other relevant clinical measures. However a recurring theme across all disciplines attending the BIDS DBS workshop in September 2010 was the importance of broad outcome measures in DBS to measure and value attributes of importance beyond those of pure clinical relevance. Broader, and more complex outcomes of DBS such as happiness, dignity, personality change, depression, euphoria, social stigma and so on may not be adequately captured within clinical measures and may need to rely on broader economic measures to "value" them. If a health service such as the provision of DBS contributes positively to human wellbeing, it has economic value. Whether something contributes to an individual's wellbeing is determined by whether or not it satisfies that individual's preferences. The basic value judgment underlying economic valuation is that "preferences count," although this does not imply that all decisions must be made on the basis of what people want. Other factors, such as what is morally appropriate, what is ethically acceptable, and what is reasonable and practical, should be taken into account, although often such factors are less amenable to formal economic analysis. Such a concept is crucially important in the economic evaluation of DBS as there are a number of ethical, moral, social, and legal factors which all play a part in this technology (as outlined by the other papers in this special edition). One way of estimating the economic 
values attached to non-marketed goods and services is to use a stated preference (SP) approach. SP approaches are based on hypothetical or constructed markets, i.e., they ask people to state what economic value they attach to attributes of those goods and services. SP methods in the DBS context would reply on health-care researchers devising questions about the risks and benefits involved in DBS and asking patients or members of the population to make valuations of the possible outcomes using money (willingness to pay methods) or identify trade-offs related to life expectancy (time-trade off methods) or risk of death (standard gamble methods). It is this approach which will be relevant to the broad evaluation of economic outcomes in DBS as it would permit the valuation of attributes beyond only health of importance to patients, as outlined above. In the area of DBS it may be that an economic SP measure may afford the measurement and valuation of a much broader range of benefits than individual clinical specific or disease specific measures are able to.

\section{DBS AND THE CAPABILITY APPROACH}

One developing approach in health economics that may be suited to such broader evaluation of DBS outcomes is the capability approach (Sen, 1993). As outlined more recently by Coast et al. (2008b) the capability approach advocates the evaluation of programs or interventions on the basis of the extent to which a person is able (has the capability) to function in a particular way. Given this, the capability approach offers a potentially much richer set of dimensions for evaluation. Nussbaum (2003) has drawn up a list of 10 central human capabilities comprising life, bodily health, bodily integrity, senses, imagination and thought, emotions, practical reason, affiliation, other species, play and control over one's environment. Indeed research using capabilities to develop a measure of outcome relevant to older people in the UK (ICECAP-O) looks promising as it contains attributes of direct relevance to the older population such as attachment, security, role, enjoyment, and control (Grewal et al., 2006; Coast et al., 2008a). The capabilities approach may be pertinent to DBS surgery in many areas as there are broader impacts than simply "health" per se with such surgery. The human capabilities outlined above such as bodily integrity, emotions, and control over one's environment can easily be linked to say the outcomes from DBS

\section{REFERENCES}

Bowling, A. (1991). Measuring Health: A Review of Quality of Life Measurement Scales. Buckingham: Open University Press.

Bowling, A. (1995). Measuring Disease. Buckingham: Open University Press.

Briggs, A. H. (2001). "Handling uncertainty in economic evaluation and presenting the results," in Economic Evaluation in Health Care: Merging Theory with Practice, eds M. F. Drummond and A. McGuire (Oxford: Oxford University Press), 172-214.

Brouwer, W., Rutten, F., and Koopmanschap, M. A. (2001). "Costing in economic evaluations," in Economic Evalaution in Health Care: Merging Theory with Practice, eds M. F. Drummond and A. McGuire

in advanced PD. Further development of such approaches will likely require the use of economic evaluation frameworks beyond cost-effectiveness and cost-utility and into the realms of costbenefit analysis (McIntosh et al., 2010).

\section{DISCUSSION}

In providing a perspective on the economic evaluation of DBS this article has attempted to cover the key topics of specific economic relevance to DBS pertinent to all indications. These key topics include the importance of appropriate handling of DBS equipment costs, the significance of evaluating within the appropriate time horizon due to the possibility of repeat DBS procedures, adverse effects, and economic impacts upon medication use affected by DBS. In addition to this the article has outlined the importance of adopting the use of broader outcome measures to allow the true impact of the wide range of effects of DBS to be valued by patients and their carers. It may be the case that newer developments such as the ICECAP-O measure or other approaches utilizing the capabilities approach capture the value of attributes of outcomes otherwise left unvalued by other measures.

The use of DBS is gradually increasing in a number of different health-care indications. While the evidence of health benefits from trials are encouraging and there have been significant QOL increases shown in DBS patients it is crucial that economic evidence complement these data so as to show the true economic impact of the technique. Key to the estimation of cost-effectiveness in the area of DBS however are the importance of unbiased long-term costs and benefit data such that the high "up front" costs of DBS are accounted for but also included are any longer term resource use implications such as IPG replacement, adverse events, and impacts upon medication use. In addition to this are the methodological challenges involved in the valuation of a broader set of benefits than have been previously accounted for. It is the belief that for many DBS applications the estimation of such broad benefits alongside long-term cost data may be the key to revealing the true economic potential of this technology.

\section{ACKNOWLEDGMENT}

This research was funded by Parkinson's UK.

and provision. Oxf. Rev. Econ. Pol. 5, 34-58. 68-93.

Buckingham, K. (1993). A note on HYE (Healthy Years Equivalents). J. Health Econ. 12, 301-309.

Buckingham, K. (1995). Economics, health and health economics - HYEs versus QALYs-a response. J. Health Econ. 14, 397-398.

Coast, J., Flynn, T., Natarajan, L., Sproston, K., Lewis, J., and Jea, L. J. (2008a). Valuing the ICECAP capability index for older people. Soc. Sci. Med. 67, 874-882.

Coast, J., Smith, R., and Lorgelly, P. (2008b). Should the capability approach be applied in health economics? Health Econ. 17, 667-670.

Culyer, A. J. (1989). The normative economics of health care finance
De Meijer, C., Brouwer, W., Koopmanschap, M., Van den berg, B., and Van Exel, J. (2010). The value of informal care-a further investigation of the feasibility of contingent valuation in informal caregivers. Health Econ.19, 755-771.

Dolan, P., Gudex, C., Kind, P., and Williams, A. (1995). A Social Tariff for EuroQol: Results from a UK General Population Survey. York: University of York.

Drummond, M. F., Sculpher, M. J., Torrance, G. W., O’Brien, B., and Stoddart, G. L. (2005). Methods for the Economic Evaluation of Health Care Programmes. Oxford: Oxford University Press.

Drummond, M. F., Stoddard, G. L., and Torrance, W. (1987). Methods for the Economic Evaluation of Health Care Programmes. Oxford: Oxford University Press.

Glick, H., Doshi, J. A., Sonnad, S. S., and Polsky, D. (2007). Economic Evaluation in Clinical Trials. Oxford: Oxford University Press.

Gold, M. R., Siegel, J. E., Russell, L. B., and Weinstein, M. C. (1996). Costeffectiveness in Health and Medicine. New York: Oxford University Press.

Grewal, I., Lewis, J., Flynn, T., Brown, J., Bond, J., and Coast, J. (2006). Developing attributes for a generic quality of life measure for older people: preferences or capabilities? Soc. Sci. Med. 62, 1891-1901.

HM Treasury (2003). The Green Book: Appraisal and Evaluation in Central Government, London: TSO, UK. 
Hoehn, M., and Yahr, M. (1967). Parkinsonism: onset, progression and mortality. Neurology 17 , 427-442.

Jenkinson, C., Fitzpatrick, R., Peto, V., Harris, R., and Saunders, P. (2008). The Parkinson's Disease Questionnaire PDQ-39 User Manual. Oxford: University of Oxford.

Joint Formulary Committee. (2010) British National Formulary, 60 Edn. London: British Medical Association and Royal Pharmaceutical Society.

Joint, C., Nandi, D., Parkin, S., Gregory, R., and Aziz, T. (2002). Hardwarerelated problems of deep brain stimulation in 100 consecutive electrodes. Mov. Disord. 17(Suppl. 3), S175-S180.

Koopmanschap, M. A., Rutten, F., van Ineveld, B. M., and van Roijen, L. (1997). The friction cost method for measuring indirect costs of disease. J. Health Econ. 6, 253-259.

McIntosh, E., Clarke, P., Frew, E., and Louviere, J. J. (2010). Applied Methods of Cost Benefit Analysis in Health Care. Oxford: Oxford University Press.

Movement Disorders Centre UoF. (2011). Movement Disorders Centre (MDC).
Nussbaum, M. (2003). Capabilities as fundamental entitlements: Sen and social justice. Fem. Econ. 9, 33-59.

Pritchard, C., and Sculpher, M. (2000). Productivity Costs: Principles and Practice in Economic Evaluation. London: Office of Health Economics.

Ramzi, D. W., and Leeper, K. V. (2004). DVT and pulmonary embolism: Part II. Treatment and prevention. Am. Fam. Physician 69, 2841-2848.

Rice, D. P., and Cooper, B. S. (1967). The economic value of human life. Am. J. Public Health 57, 1954-1966.

Richardson, J. (1994). Cost utility analysis - what should be measured? Soc. Sci. Med. 39, 7-21.

Sackett, D. L., and Torrance, G. W. (1978). The utility of different health states as perceived by the general public. J. Chronic Dis. 31, 697-704.

Sen, A. (1993). "Capability and wellbeing," in The Quality of Life, eds M. Nussbaum and A. sen (Oxford: Clarendon Press), 30-53.

The EuroQol Group. (2011).EQ-5D Home Page. Available at: www.euroqol.org

Torrance, G. W. (1976). Social preferences for health states: an empirical evalua- tion of three measurement techniques. Socioecon. Plan. Sci. 10, 128-136.

Torrance, G. W., Boyle, M. H., and Horwood, S. P. (1982). Application of multiattribute utility theory to measure social preferences for health states. Oper. Res. 30, 1043-1069.

Torrance, G. W., and Sackett, D. L. (1972). A utility maximising model for evaluation of health care programmes. Health Serv. Res. 7, 118-133.

Van Buuren, S., Boshuizen, H. C., and Knook, D. L. (1999). Multiple imputation of missing blood pressure covariates in survival analysis. Stat. Med. 18 681-694.

Van den berg, B., and Ferrer-I-Cabonell, A. (2007). Monetary valuation of informal care: the well-being valuation method. Health Econ. 16, 1227-1244.

Van Houtven, C. N., and Norton, E. C. (2004). Informal care and health care use of older adults. J. Health Econ. 23 , 1159-1180.

Williams, A. (1985). Economics of coronary artery bypass grafting. $\mathrm{Br} \mathrm{Med} J$ 291, 326-329.

Williams, A., Gill, S., varma, T. Jenkinson, C., Quinn, N., mitchell, R., Scott, R., Ives, N., Rick, C.,
Daniels, J., Patel, S., Wheatley, K., and PD SURG Collaborative Group. (2010). Deep brain stimulation plus best medical therapy versus best medical therapy alone for advanced Parkinson's Disease (PDSURG Trial): a randomised, open-label trial. Lancet Neurol. 9, 581-591.

Conflict of Interest Statement: The author declares that the research was conducted in the absence of any commercial or financial relationships that could be construed as a potential conflict of interest.

Received: 11 January 2011; accepted: 04 May 2011; published online: 16 May 2011. Citation: McIntosh ES (2011) Perspective on the economic evaluation of deep brain stimulation. Front. Integr. Neurosci. 5:19. doi: 10.3389/fnint.2011.00019

Copyright (c) 2011 McIntosh. This is an open-access article subject to a nonexclusive license between the authors and Frontiers Media SA, which permits use, distribution and reproduction in other forums, provided the original authors and source are credited and other Frontiers conditions are complied with. 